\title{
About mycoplasma infection
}

\section{Takeo Fuse*}

Department of Otorhinolaryngology, Prefectural Kahoku Hospital, Kahoku-cho, Yamagata, 999-35, Japan

Nasopharyngeal findings are important for the diagnosis and treatment of mycoplasma infection because the diagnosis of this infection is conducted by sputum sampling using Ribotest. By differential diagnosis of nasopharyngeal masses using a fiber rhino laryngoscope, it is possible to ascertain purulent nasal discharge as a characteristic of sinusitis and viscous sputum as a characteristic of mycoplasma. It is important to establish the specific symptoms and nasopharyngeal findings to diagnose a mycoplasma infection.

Mycoplasma is characterized by a unique cough that presents itself as a bad cough that lingers all the time and makes it very difficult for the affected person to sleep. When these symptoms are evident, there is a high possibility of mycoplasma infection. In addition, it is crucial to observe whether parents opt for a strong or weak cough remedy. Parents opting for a strong cough remedy might be suffering from the cough of their children. In many cases, mycoplasma presents itself as a gradually worsening cough after fever and sore throat, but such symptoms could also be those of rhinorrhea or sore throat only. Rhinorrhea or sore throat is preceded, and sometimes followed, by fever, which may last for several days, but often only for one day. There is sudden high fever and body fatigue, similar to influenza. Therefore, if it occurs in a season known for the spread of influenza, it is difficult to identify the infection correctly.

Although nasopharyngeal findings can help in the diagnosis of mycoplasma, it is important to note that the viscous sputum is collected on the rear wall of the nasopharynx with a smooth nasal discharge, which makes it impossible to observe directly from the oral cavity. However, this finding is discernable at an early stage of the infection (1-2 days), and it depends on whether the age of the patient is suitable for a specific line of treatment. For example, if the patient is below2-3 years old, it is not possible to advise the patient to blow the nose. In such cases, it is required to first suck the nasal tract with an olive tube and then observe the area by using a fiber rhino laryngoscope. In the current study, I used a fiber rhino laryngoscope manufactured by Olympus Corporation. This equipment is much better than electronic scopes, which may be useful for use in large hospitals but are too cumbersome for clinical use.

It must be noted here that mycoplasma with only sore throat does not have redness of the palatal tonsils, the laryngeal mucosa is somewhat red, and the nasopharyngeal fiber appears as a membrane in the thin sputum. Where the thickest sputum is the thickest is decided. In case of patients under 6 years old, the nasopharynx is narrow and can be composed of screens, but it is important to investigate by correlating the symptoms with patient interactions and other local factors. A right-handed specialist needs to hold the swab in the right hand and use the left to push the tongue downward with the help of a tongue indenter. The first step is to place one end of the cotton swab in the nasopharynx and remove the sputum of the nasopharynx by rotating the cotton swab to the right, from the left side of the patient. During the collection process, there is a high possibility of mycoplasma when white viscous phlegm is visible on the right side of the patient's uvula. In the case of patient older than 6 years, the nasopharynx is wide, and fiber is used to check the position of the sputum. If the sputum is above the nasopharynx, it is required to pull the cotton swab upward with the tongue indenter and collect the sputum. In case of acute sinusitis, the sputum is gathered in the middle nasal passage for a while after careful aspiration of purulent rhinorrhea, but mycoplasma does not have such outcomes. Nasal washing by raw eating is effective for purulent posterior nasal discharge in the case of acute sinusitis in adults. In the case of children with posterior nasal discharge, gargling with not only nasal aspiration but also aspiration of the nasal congestion of the nasopharynx from the oral cavity is necessary. In addition, if there is no indicative finding on the nasopharynx, as in the case of a cough caused by allergy, meptin inhalation could help differentiate the cause of the cough.

For treatment of patients below 7 years old, TFLX is administered for a minimum of 4 days. Fever drops within 4 days at the most. When TFLX is administered for 4-7 days, the Ribotest is almost always negative. For children who cannot take TFLX, administer CLDM after explaining its bitterness. The therapeutic effect of the treatment is ascertained from clinical symptoms and nasopharyngeal findings. If an antibiotic is effective, the sputum of the nasopharynx gradually decreases while smooth rhinorrhea remains intact. In case inflammatory symptoms persist, it would be necessary to add CAM in the treatment. In cases where the patient is unable to blow the nose, the rhinorrhea becomes dry, but becomes purulent as it gets worse. If there is no improvement even after administering TFLX for 7 days, it might be advisable to opt for the intravenous injection of CLDM. Although macrolide is used for the treatment of sinusitis, there are cases where mycoplasma is affected by macrolide and initial administration of CAM is not recommended. In addition, consumption of only CAM for 10 days may consolidate the bacteria even if symptoms improve. Purulent nasal discharge, above or in middle meatus of acute sinusitis, decreases with 4-8 daily doses of penicillin and cephem. If it still persists, CAM4_nichikan can help eliminate it completely. In such cases, sucking the purulent posterior nasal drainage with a thin aspiration tube is an important line of treatment to alleviate lack of sleep due to nasal congestion. Dosage of MINO is advised for patients aged 8 years, although this age grouping might be subject to fluctuations. A dosage of $3-4 \mathrm{mg} / \mathrm{kg}$ tablets in the mornings and evenings is advised

*Correspondence to: Takeo Fuse, Department of Otorhinolaryngology, Prefectural Kahoku Hospital, Kahoku-cho, Yamagata, 999-35, Japan, E-mail: musume@ ma.catvy.ne.jp

Received: January 23, 2019; Accepted: February 02, 2019; Published: February 04,2019 
for 2 for 4 days. MINO usually takes effect for several hours, but there are cases where fever occurs in the night when MINO is administered at about 1 in the morning. Therefore, it is not recommended. After administering MINO for 4-8 days, the Ribotest is almost negative. If MINO is ineffective, TFLX needs to be administered to most children. Even though symptoms settle down after 4 days of antibiotic treatment, cold symptoms without fever were observed two days later. However, it is not a relapse of mycoplasma. For cases with severe cough, codeine is administered, and steroid administration might be required for cases with severe respiratory distress. For patients over the age of 15 years, LVFX is orally administered to suspected cases of mycoplasma based on interviews and local factors. For pregnant and lactating women, CAM is administered for up to 10 days after carefully examining such cases and emphasizing safety precautions. If LVFX is not effective, $600 \mathrm{mg}$ of CLDM could be administered by drip infusion in cases of confirmed mycoplasma infection. If oral intake is changed to MINO, the symptoms will improve and the Ribotest will be negative. LVFX should be effective for mycoplasma, but in case of no improvement even after 8 days of intake, the Ribotest will be positive. If cough is severe, administer Decadron $(2 \mathrm{mg})$. Intravenous administration of CLDM has an immediate effect on mycoplasma, and that can help confirm the infection. In case the Ribotest negative, blood is collected, and leukocytes and CRP elevated, it could be a bacterial infection other than mycoplasma, and it is necessary to administer a penicillin antibiotic. In mycoplasma infection, in principle leukocytes and CRP are normal.

The accuracy of the Ribotest is $70 \%$, and when similar symptoms are found in the family, antibiotics are administered for mycoplasma infection. The incubation period of mycoplasmas is 2-3 days, and the shortest period of reinfection is about 2 weeks. If you believe that the cough is finally cured, reinfection may still occur with fever. Because the immune function is developed in adults, the bacteria might disappear as result of the antibiotics, but coughing may last for more than three months in severe cases. In such cases, viscous sputum gradually converts to normal foamy sputum and disappears 7 to 10 days after administration of antibiotics. Since cough continues, chest X-ray is recommended to exclude lung cancer and tuberculosis. In addition, the infection damages the airway mucosa itself, cough is easily induced because of changes in temperature and other similar factors. If the patient doesn't slip into a serious condition, such as pneumonia, because of lack of antibiotic treatment, the severe cough is at a peak for 2 weeks, after which it gradually goes down and becomes mild after 1 month. In the case of only sore throat, following severe body fatigue for about 2-3 days, a cure can be expected in about 1 week. However, in the case of mycoplasma infection, viscous sputum of the nasopharynx continues and is often asymptomatic in young people, but in the elderly, it is necessary to differentiate it from reflux esophagitis, where the patient complains of pharynx discomfort and foreign body sensation. This settled condition may last for several months and the Ribotest is positive for the viscous sputum of the pharynx. However, the symptoms and phlegm are eliminated by administration of appropriate antibiotics.

Over the past two years, the number of cases with a positive Ribotest in our department is 1585 including duplication patients. 32in September $2015 \cdot 38$ in October $\cdot 53$ in November $\cdot 59$ in December .54 in January $2016 \cdot 88$ in February 98 in March 52 in April . 75 in May .68 in June. 48 in July. 21 in August .38 in September .89 in October . 92 in November 93 in December 111 in January 2017.91 in February . 149 in March . 72 in April . 84 in May · 57 in June · 45 in July · 32 in August. LVFX is administered to adults suspected of mycoplasma infection, but antibiotics effective for acute sinusitis and mycoplasma are different in the case of children. Therefore, most of the positive cases in our studywere under 15 years old, with a minimum age of 3 months. Based on the nasopharyngeal findings, the probability of being positive for mycoplasma was $80-90 \%$. Initially, I was convinced about mycoplasma from the symptoms and nasopharyngeal findings but did not prescribe antibiotics for cases that were negative in the examination. After a few days of such cases, positive cases of families followed, and antibiotics were prescribed. Although Ribotest is a revolutionary diagnostic method, it has a high frequency of false positives and false negatives, and there is also a problem of settled status. Therefore, observation of clinical symptoms and nasopharyngeal findings was considered indispensable for diagnosing mycoplasma and deciding the line of treatment.

If mycoplasma infection is presented with only sore throat and no difference in the left / right sides, the diagnosis of so-called acute pharyngitis will be meaningless. Mycoplasma may be a disease originating from the lower respiratory tract, but it would be better to consider it as an infection with systemic symptoms similar to those of influenza. Also, viruses such as influenza cannot be contained easily and spread to other humans, which results in expansion and convergence of the virus. On the other hand, bacteria such as mycoplasma have a condition of settling down, and attacks are possible all year round. Many viruses that cause so-called cold symptoms, such as respiratory syncytial virus (RSV) and adenovirus, can be immunized after 6 years of age and are unlikely to become severe Considered as a whole, this means that cases redness or nasal pharynx of palatine tonsil with inflammation findings, such as purulent post-nasal discharge and viscous phlegm and nasal pharyngeal wall mucosa of redness, are likely to be EB or influenza and they may be mostly a bacterial infection. It may be noted that in cold-like symptoms accompanying gastroenteritis, the findings of the nasopharynx are normal. A mild cold with symptoms that do not require antibiotics might be inflamed with minor nasopharyngeal findings, but it is cured in 3-4 days. Normally, this mild cold does not reach our department, but when influenza type B becomes prevalent, the number of arrivals of worried parents with their children increases.

Copyright: (C2019 Fuse T. This is an open-access article distributed under the terms of the Creative Commons Attribution License, which permits unrestricted use, distribution, and reproduction in any medium, provided the original author and source are credited. 